\title{
Applications of the Schwinger multichannel method to electron-molecule collisions
}

\author{
Marco A. P. Lima, Luiz M. Brescansin, and Antônio J. R. da Silva \\ Instituto de Física Gleb Wataghin, Unicamp, Caixa Postal 6165, 13081 Campinas, Sao Paulo, Brazil \\ Carl Winstead and Vincent McKoy \\ Noyes Laboratory of Chemical Physics, California Institute of Technology, Pasadena, California 91125
}

(Received 24 July 1989)

\begin{abstract}
We discuss some recent developments in the implementation of the Schwinger multichannel method for electron-molecule collision calculations. The evaluation of matrix elements involving the operator $V G_{P}^{(+)} V$, previously accomplished by insertion of a Gaussian basis on either side of $G_{P}^{(+)}$, is now done by direct numerical quadrature. This approach avoids the necessity of very large Gaussian basis sets, allowing the size of the basis to reflect only the dynamical requirements of the scattering wave function. We find that the reduction in the required basis size results in improved efficiency, in spite of the additional numerical effort of performing the quadrature. Trial applications to electron- $\mathrm{CH}_{4}$ scattering in the static-exchange approximation and to electronic excitation of $\mathrm{H}_{2}$ illustrate the excellent convergence characteristics of the procedure.
\end{abstract}

\section{INTRODUCTION}

In recent years the Schwinger multichannel (SMC) method $^{1-3}$ has been used to study the cross sections for elastic scattering of low-energy electrons by diatomic ${ }^{4,5}$ and polyatomic ${ }^{6-10}$ molecules and for electron-impact excitation of $\mathbf{H}_{2}$ (Refs. 11 and 12). The SMC method allows for an expansion of the complete scattering wave function in square-integrable basis sets. ${ }^{1}$ With an expansion of this wave function in Cartesian Gaussians all spatial integrations arising in our procedure for the evaluation of matrix elements in the associated variational expression can be done analytically for arbitrary molecular targets. ${ }^{1}$ This feature has obvious practical consequences in applications of the method to nonlinear polyatomics. In applications to date $\mathrm{e}^{4-11}$ we have inserted approximate unit operators defined on Cartesian Gaussian bases around the Green's function to avoid the numerical integrations over linear momentum variables arising in the principal-value piece of the $V G_{P}^{(+)} V$ matrix elements. ${ }^{4-10}$ In applications of the method, however, this insertion technique generally required far more basis functions than could be expected to be needed to approximate the scattering wave function well in Schwinger-like variational methods. ${ }^{13}$ Furthermore, the results of several studies using a fixed scattering basis of modest size and different insertion sets of increasing size were disappointing. In general, we found the procedure of using the same very large basis for both scattering and insertion to be the most reliable. For example, convergence of the elastic cross sections for systems such as $\mathrm{H}_{2} \mathrm{O}, \mathrm{NH}_{3}$, and $\mathrm{CH}_{4}$ required of the order of 80 to 90 uncontracted Cartesian Gaussian functions, ${ }^{6,8,9}$ while 64 such functions were used in two-channel calculations of the electronic excitation of $\mathrm{H}_{2}$ (Ref. 11). However, two-channel studies of electron-impact excitation of $\mathrm{CO}$ revealed that convergence with this many functions was still questionable and suggested that further increases in the basis would be im- practical. ${ }^{14}$ Furthermore, in multichannel studies of electronic excitation of the simpler system $\mathrm{H}_{2}$, the opening of additional electronic channels introduced effects in our cross sections that seemed to be multichannel, while in fact they could just reflect a lack of convergence in the basis.

In this paper we present results of applications of the SMC method in which this insertion procedure is avoided and the integrations over linear momentum variables arising in the $V G_{P}^{(+)} V$ matrix elements are performed by numerical quadrature. This procedure will be seen to improve the efficiency of the calculations significantly by reducing the size of the Gaussian basis sets needed to obtain reliable cross sections. The size and composition of these basis sets now reflect the dynamical requirements of the scattering wave function only. In this way the physically uninteresting requirements of the insertion technique, which led to the use of very large basis sets, are disentangled from the dynamical needs of the collision. We illustrate this new approach with applications to elastic scattering of electrons by $\mathrm{CH}_{4}$ in the static-exchange approximation and to electron-impact excitation of $\mathrm{H}_{2}$. Comparison between the present calculations with quite modest basis sets and earlier studies with large basis sets indicates that excellent convergence is obtained. This behavior now more closely reflects the known convergence characteristics of Schwinger-like variational methods.

\section{FORMULATION}

Details of the SMC method have been discussed extensively elsewhere. ${ }^{1-11}$ Here we will review a few steps in the development which are essential to the present discussion.

The Hamiltonian for the $(N+1)$-electron collision system can be written as

$$
H=\left(H_{N}+T_{N+1}\right)+V=H_{0}+V
$$


where $H_{N}$ is the target Hamiltonian, $T_{N+1}$ is the kinetic energy operator for the incident electron, and $V$ is the interaction between the scattering electron and the nuclei and electrons of the target. The complete scattering wave function can be shown to satisfy a modified Lippmann-Schwinger equation ${ }^{1}$

$$
A^{(+)} \Psi_{\mathbf{k}_{m}}^{(+)}=V S_{\mathbf{k}_{m}},
$$

where

$$
\begin{aligned}
A^{(+)}= & \frac{1}{2}(P V+V P)+\frac{1}{N+1}\left(\hat{H}-\frac{N+1}{2}(\hat{H} P+P \hat{H})\right) \\
& -V G_{P}^{(+)} V,
\end{aligned}
$$

$S_{\mathbf{k}_{m}}=\Phi_{m}(1, \ldots, N) e^{i \mathbf{k}_{m} \cdot \mathbf{r} N+1}$ are solutions of the unperturbed Hamiltonian $H_{0}$, and $\hat{H}=E-H . P$ is a projection operator defined on the open-channel eigenstates $\Phi_{l}$ of $H_{N}$, and $G_{P}^{(+)}$is the projected outgoing-wave Green's function defined formally as

$$
\begin{aligned}
G_{P}^{(+)} & =P\left(E-H_{0}+i \epsilon\right)^{-1} \\
& =\sum_{l \in \text { open }} \frac{\left|\Phi_{l}\right\rangle\left\langle\Phi_{l}\right|}{\frac{1}{2} k_{l}^{2}-T_{N+1}+i \epsilon},
\end{aligned}
$$

where $\frac{1}{2} k_{l}^{2}$ is the electron kinetic energy associated with the $\Phi_{l}$ channel. $G_{P}^{(+)}$can be written as

$$
G_{P}^{(+)}=\sum_{l \in \text { open }} \int d^{3} k \frac{\left|\Phi_{l} \mathbf{k}\right\rangle\left\langle\mathbf{k} \Phi_{l}\right|}{\frac{1}{2} k_{l}^{2}-\frac{1}{2} k^{2}+i \epsilon}
$$

and separated into a residue and principal-value term

$$
G_{P}^{(+)}=G_{P}^{R}+G_{P}^{P},
$$

where

$$
G_{P}^{R}=i \pi \sum_{l \in \text { open }} k_{l} \int d \Omega_{\mathbf{k}_{l}}\left|\Phi_{l} \mathbf{k}_{l}\right\rangle\left\langle\mathbf{k}_{l} \Phi_{l}\right|
$$

and

$$
G_{P}^{P}=\sum_{l \in \text { open }} \mathbf{P} \int_{0}^{\infty} d k \frac{k^{2}}{\frac{1}{2} k_{l}^{2}-\frac{1}{2} k^{2}} \int d \Omega_{\mathbf{k}}\left|\Phi_{l} \mathbf{k}\right\rangle\left\langle\mathbf{k} \Phi_{l}\right| .
$$

In Eq. (6c) $P$ represents the Cauchy principal value.

Based on the inhomogeneous Eq. (2), a multichannel variational expression for the scattering amplitude is given by

$$
\begin{aligned}
f_{\mathbf{k}_{f} \mathbf{k}_{i}}=-\frac{1}{2 \pi} & \left(\left\langle S_{\mathbf{k}_{f}}|V| \Psi_{\mathbf{k}_{t}}^{(+)}\right\rangle+\left\langle\Psi_{\mathbf{k}_{f}}^{(-)}|V| S_{\mathbf{k}_{t}}\right\rangle\right. \\
& \left.-\left\langle\Psi_{\mathbf{k}_{f}}^{(-)}\left|A^{(+)}\right| \Psi_{\mathbf{k}_{i}}^{(+)}\right\rangle\right) .
\end{aligned}
$$

Expansion of $\Psi_{\mathbf{k}}^{( \pm)}$in terms of Slater determinants

$$
\Psi_{\mathbf{k}}^{( \pm)}=\sum_{m} a_{m}^{( \pm)}(\mathbf{k}) \Psi_{m}
$$

and the requirement of stability with respect to variation of the coefficients $a_{m}^{( \pm)}(\mathbf{k})$ leads to

$f_{\mathbf{k}_{f} \mathbf{k}_{i}}=-\frac{1}{2 \pi} \sum_{m n}\left\langle S_{\mathbf{k}_{f}}|V| \Psi_{m}\right\rangle\left(d^{-1}\right)_{m n}\left\langle\Psi_{n}|V| S_{\mathbf{k}_{l}}\right\rangle$, where

$$
d_{m n}=\left\langle\Psi_{m}\left|A^{(+)}\right| \Psi_{n}\right\rangle .
$$

If the orbitals in the $(N+1)$-electron Slater determinants are expanded in Cartesian Gaussian functions, all matrix elements arising in Eq. (9) can be evaluated analytically with the important exception of those from $\left\langle\Psi_{m}\left|V G_{P}^{(+)} V\right| \Psi_{n}\right\rangle$. Using Eqs. (6b) and (6c), these matrix elements can be written as

$$
\begin{aligned}
\left\langle\Psi_{m}\left|V G_{p}^{(+)} V\right| \Psi_{n}\right\rangle= & \left\langle\Psi_{m}\left|V G_{P}^{R} V\right| \Psi_{n}\right\rangle \\
& +\left\langle\Psi_{m}\left|V G_{P}^{P} V\right| \Psi_{n}\right\rangle,
\end{aligned}
$$

where

$$
\left\langle\Psi_{m}\left|V G_{P}^{R} V\right| \Psi_{n}\right\rangle=i \pi \sum_{l \in \text { open }} k_{l} g_{m n}^{l}\left(k_{l}\right)
$$

and

$$
\left\langle\Psi_{m}\left|V G_{P}^{P} V\right| \Psi_{n}\right\rangle=\sum_{l \in \text { open }} \mathrm{P} \int_{0}^{\infty} d k \frac{k^{2}}{\frac{1}{2} k_{l}^{2}-\frac{1}{2} k^{2}} g_{m n}^{l}(k),
$$

with

$$
g_{m n}^{l}(k)=\int d \Omega_{\mathbf{k}}\left\langle\Psi_{m}|V| \Phi_{l} \mathbf{k}\right\rangle\left\langle\mathbf{k} \Phi_{l}|V| \Psi_{n}\right\rangle .
$$

Takatsuka and McKoy ${ }^{1}$ originally proposed an insertionlike quadrature for evaluation of the matrix elements in Eqs. (12a) and (12b). This procedure, referred to as the $\alpha$-insertion technique, essentially consists of constructing an approximate unit operator out of a large number of Cartesian Gaussians, i.e., $\mathbb{1} \approx \sum_{\alpha}|\alpha\rangle\langle\alpha|$. Insertion of this operator in Eqs. (12a) and (12b) leads to

$$
\begin{aligned}
\left\langle\Psi_{m}\left|V G_{P}^{R} V\right| \Psi_{n}\right\rangle=i \pi \sum_{l \in \text { open }} \sum_{\alpha \beta} k_{l}\left\langle\Psi_{m}|V| \Phi_{l} \alpha\right\rangle \\
\times\left\langle\beta \Phi_{l}|V| \Psi_{n}\right\rangle h_{\alpha \beta}\left(k_{l}\right)
\end{aligned}
$$

and

$$
\begin{aligned}
&\left\langle\Psi_{m}\left|V G_{P}^{P} V\right| \Psi_{n}\right\rangle=\sum_{l \in \text { open } \alpha \beta} \sum_{m}\left\langle\Psi_{m}|V| \Phi_{l} \alpha\right\rangle\left\langle\beta \Phi_{l}|V| \Psi_{n}\right\rangle \\
& \times P \int_{0}^{\infty} d k \frac{k^{2}}{\frac{1}{2} k_{l}^{2}-\frac{1}{2} k^{2}} \\
& \times h_{\alpha \beta}(k)
\end{aligned}
$$

where

$$
h_{\alpha \beta}(k)=\int d \Omega_{\mathbf{k}}\langle\alpha \mid \mathbf{k}\rangle\langle\mathbf{k} \mid \beta\rangle .
$$

The function $h_{\alpha \beta}(k)$ can be obtained analytically, and the principal-value integral in Eq. (12b) can be evaluated in closed form. ${ }^{15}$ Equations (13b) and (13c) show that this procedure relies on a spectral decomposition of $|\mathbf{k}\rangle$ onto a finite Gaussian basis. If the matrix element in Eq. (13b) is important in an application of interest, this decomposition of $|\mathbf{k}\rangle$ must be adequate for a potentially wide range of $|\mathbf{k}|$. This is a formidable requirement for a basis of reasonable size. 


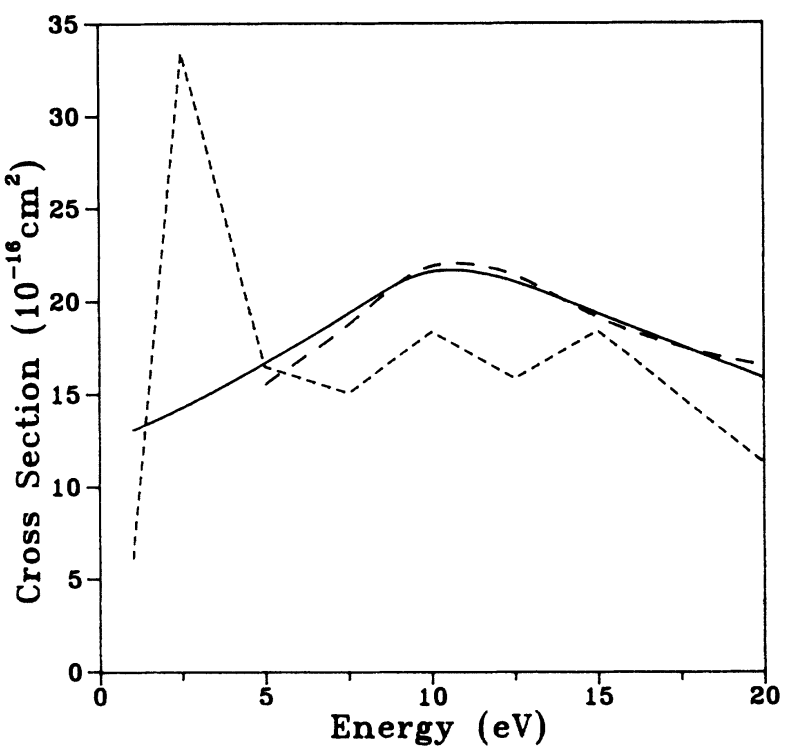

FIG. 1. Elastic electron-scattering cross section for $\mathbf{C H}_{4}$. Solid line: present results using numerical quadrature of $V G_{P}^{(+)} V$ integrals and 27 scattering functions; short dashes: $k$ insertion results in the same basis set; long dashes: converged $k$-insertion results of Ref. 6 .

Convergence of this procedure could be partially assessed by monitoring the unitarity of the $S$ matrix, which in turn depends entirely on how well the on-shell elements of Eq. (12a) are approximated by Eq. (13a)..$^{5}$ Experience showed that this procedure could require a substantial number of Gaussian functions even for elastic scattering. This led us to a hybrid procedure in which the on-shell or residue contribution $\left\langle\Psi_{m}\left|V G_{P}^{R} V\right| \Psi_{n}\right\rangle$ is evaluated by numerical quadrature, thus assuring unitarity, ${ }^{5}$ and the insertion quadrature Eq. (13b) is used to evaluate the principal-value contribution with its off-shell integrations. This hybrid technique, referred to as $k$ insertion, has been used in most applications of the SMC method to date..$^{5-11}$

In more recent applications of the SMC method to systems such as $\mathrm{N}_{2}$ and $\mathrm{CO}$ with the $k$-insertion technique, we learned that convergence of the insertion quadrature in Eq. (13b) could demand very large Gaussian basis sets. For example, convergence could still be questionable with over 90 uncontracted Cartesian Gaussians, making further attempts to assess convergence quite impractical. Furthermore, we observed that, even for simpler systems such as $\mathrm{H}_{2}$, opening of new electronic channels in a calculation could introduce effects in our cross sections that could be perceived as multichannel while, in fact, they reflected only a failure in achieving convergence in $\mathrm{Eq}$. (13b).

These considerations suggested that Eq. (13b) should be abandoned in multichannel studies, where the Green's function of Eq. (5) must be well represented for all open channels, and hence in different energy regions, simultaneously. In the next section we present the results of applications of the SMC method in which the off-shell
$V G_{P}^{(+)} V$ integrals in Eqs. (12b) and (12c) are evaluated by numerical quadrature, thus avoiding the use of any insertion quadrature. We will see that with this procedure relatively small basis sets are capable of providing reliable cross sections. We also find that, generally, the reduction in basis set size afforded by abandoning Eq. (13b) more than compensates for the additional effort required to evaluate Eq. (12b) numerically.

\section{APPLICATIONS}

In this section we test the numerical quadrature procedure for $V G_{P}^{(+)} V$ matrix elements by applying it to two molecular test cases. The first case is elastic scattering by methane in the static-exchange approximation; the second is electronic excitation of $\mathrm{H}_{2}$ to the $a^{3} \Sigma_{g}^{+}$and $b^{3} \Sigma_{u}^{+}$states in the two-channel approximation. These examples were chosen to allow assessment of the numerical quadrature technique in realistic applications for which comparison to insertion results ${ }^{6,11}$ obtained with much larger basis sets is possible. Moreover, the convergence of the insertion calculations has been confirmed by comparable theoretical studies using independent numerical methods for both the $\mathrm{CH}_{4}$ elastic ${ }^{16,17}$ and the $\mathbf{H}_{2}$ $b^{3} \Sigma_{u}^{+}$excitation ${ }^{18,19}$ cross sections. In the present work we have deliberately chosen basis sets which are too small, based on our past experience, to yield meaningful results using the $k$-insertion technique, and which therefore provide a stringent test of the numerical quadrature procedure.

\section{A. Methane}

For the present static-exchange calculations on methane we employed Dunning's ${ }^{20}(5 s, 3 p)$ set of contracted Cartesian Gaussians on $\mathrm{C}$ and $3 s$ contracted

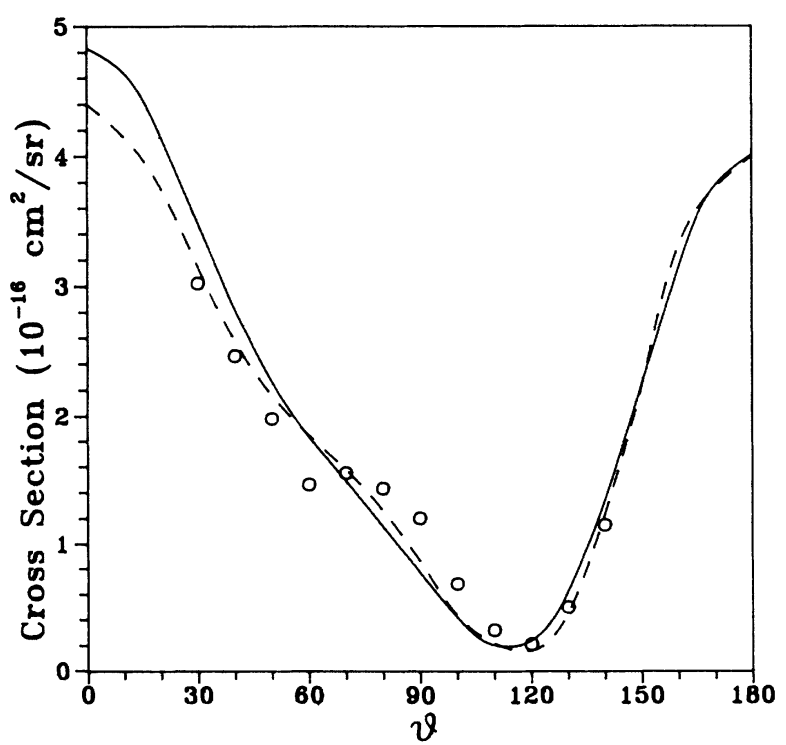

FIG. 2. Differential electron- $\mathrm{CH}_{4}$ elastic scattering cross section at $7.5 \mathrm{eV}$. Solid line: present results; dashed line: results of Ref. 6; circles: experimental results of Tanaka et al. (Ref. 21). 


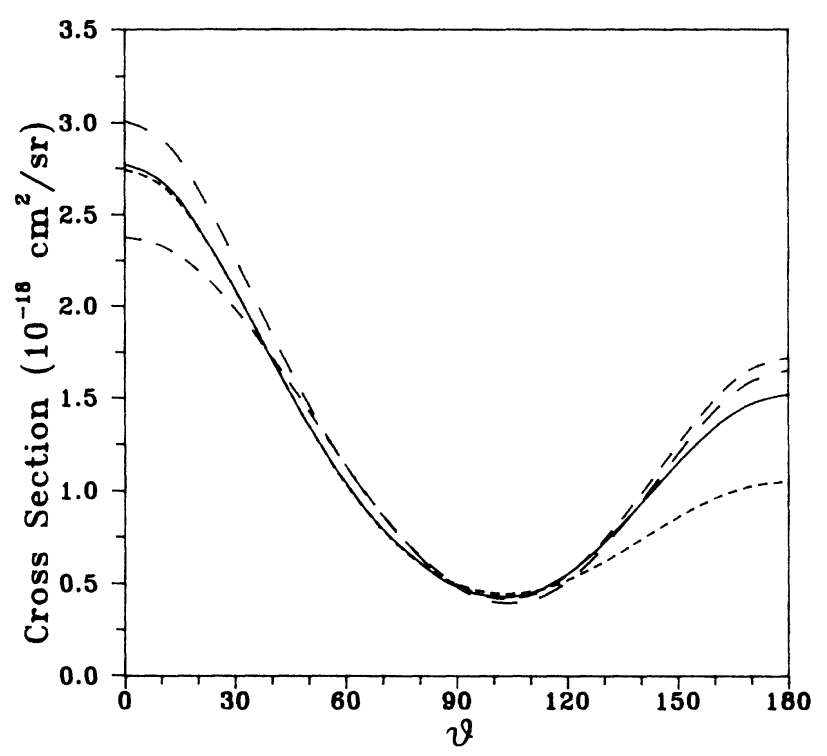

FIG. 3. Differential cross section for excitation of $\mathrm{H}_{2}$ to the $a^{3} \Sigma_{g}^{+}$state at $20 \mathrm{eV}$ impact energy. Short dashes: 17-function basis; medium dashes: 30-function basis; long dashes: 46function basis; solid line: $k$-insertion results of Ref. 11 (see text for discussion).

Gaussians on $\mathrm{H}$ for describing both the $\mathrm{CH}_{4}$ target and the scattering orbitals. In addition, we included three Cartesian $d$ Gaussians (exponents $0.32,0.128$, and 0.0512) in the expansion of the scattering orbital; however, we retained only those components of the $d$ functions which contributed to $E$ symmetry. Allowing for the five occupied orbitals, we therefore had a scattering basis of 27 functions. This basis is a subset of that used in Ref. 17 and represents both a subset and a contraction of the basis used in the earlier $k$-insertion study, ${ }^{6}$ where a total of 83 uncontracted scattering functions was employed. The present basis was chosen as being of the minimal size necessary to describe the molecular target and to allow a fair representation of the $E$-symmetry contribution to the scattering wave function.

Our results for the total elastic scattering cross section using the 27 -function basis and the numerical quadrature technique are shown in Fig. 1. Evidently, the results of this economical calculation are in excellent agreement with the insertion results of Ref. 6, which required much greater computational effort. The almost surprising performance of the method in this small basis is highly encouraging. Also included in Fig. 1, for purposes of comparison only, is the $k$-insertion result obtained from the same 27-function basis used for the numerical quadrature calculation. As we stated earlier, our past experience with the insertion method led us to expect that it would fail in so small a basis; however, the comparison in Fig. 1 serves to make explicit the point that this failure is due solely to a poor representation of the Green's function, and not to an inadequate expansion of the scattering wave function. These results suggest that the numerical quadrature procedure may permit the use of moderate basis sets in studies of more complex molecules, for which convergence of the $k$-insertion procedure, which can only be assured by repeating the calculation in progressively larger bases, quickly becomes impractical.

As might be anticipated from the agreement of the present total cross section with that of Ref. 6, the differential cross sections are also in very good accord. To give an example, we show in Fig. 2 the differential elastic scattering cross section at $7.5 \mathrm{eV}$, both as obtained from the present numerical-quadrature study and as reported in Ref. 6. This figure also shows that both calculations reproduce the experimental cross section ${ }^{21}$ very well, apart from the shoulder at $60^{\circ}-80^{\circ}$, which is known ${ }^{7}$ to be due to polarization effects omitted in both calculations. The situation at other scattering energies is highly comparable to that illustrated in Fig. 2, and consequently these results are not shown.

\section{B. $\mathbf{H}_{2}$}

The second test problem chosen for the numerical quadrature technique is inelastic scattering from $\mathrm{H}_{2}$. We have carried out two-state calculations of the differential excitation cross section at selected energies for both the $a^{3} \Sigma_{g}^{+}$and the $b^{3} \Sigma_{u}^{+}$channels using several small basis sets ranging in size from 17 to 46 scattering functions. Once again, comparison with insertion calculations ${ }^{11}$ carried out in an extensive Gaussian basis provides a point of reference for assessing the present results.

Differential cross sections for the $a^{3} \Sigma_{g}^{+}$excitation channel at 20 and $30 \mathrm{eV}$ impact energy are shown in Figs. 3 and 4 , respectively. In addition to the cross sections of Ref. 11, obtained using the insertion technique and a basis of 63 uncontracted Gaussian orbitals, we present numerical quadrature results using small, intermediate, and fairly large basis sets, containing, respectively 17,30 , and 46 contracted Gaussian scattering orbitals. The de-

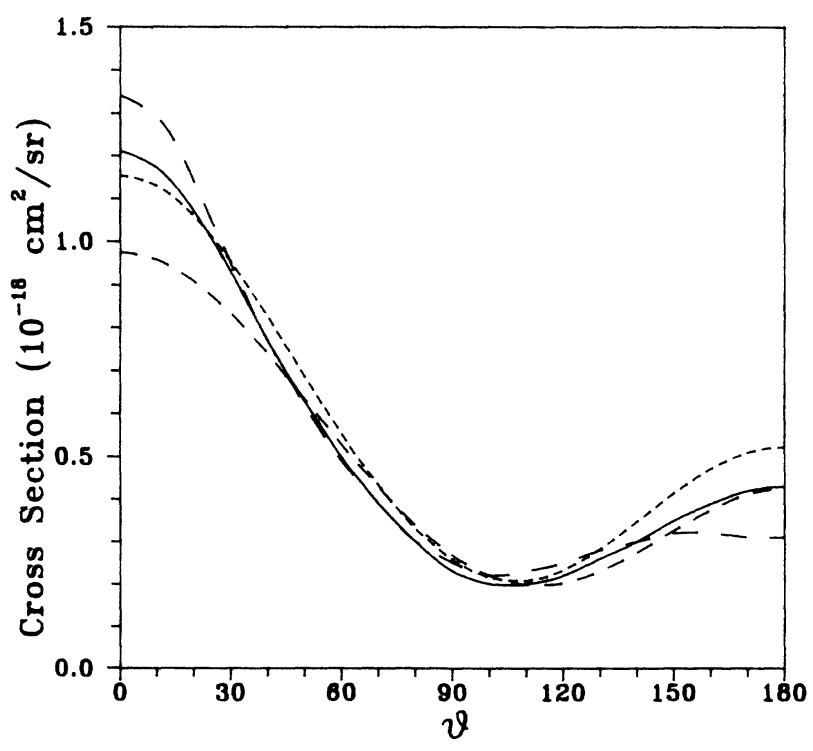

FIG. 4. As in Fig. 3, at $30 \mathrm{eV}$. 
TABLE I. Basis sets used in $\mathrm{H}_{2} a^{3} \Sigma_{g}^{+}$and $b^{3} \Sigma_{u}^{+}$calculations.

\begin{tabular}{|c|c|c|c|}
\hline Center & Function type & Exponent & Coefficient \\
\hline \multicolumn{4}{|c|}{17 scattering function basis ${ }^{a}$} \\
\hline $\mathbf{H}$ & $s$ & 0.06 & 1.0 \\
\hline $\mathbf{H}$ & $p$ & 0.48 & 1.0 \\
\hline $\mathbf{H}$ & $p_{z}$ & 0.24 & 1.0 \\
\hline c.m. & $s$ & 0.025 & 1.0 \\
\hline c.m. & $s$ & 0.01 & 1.0 \\
\hline \multicolumn{4}{|c|}{30 scattering function basis } \\
\hline $\mathbf{H}$ & $s$ & 48.4479 & 0.03294438 \\
\hline $\mathbf{H}$ & $s$ & 7.28346 & 0.240369625 \\
\hline $\mathbf{H}$ & $s$ & 1.65139 & 1.11653816 \\
\hline $\mathbf{H}$ & $s$ & 0.462447 & 2.55414162 \\
\hline $\mathbf{H}$ & $s$ & 0.145885 & 2.21455876 \\
\hline $\mathbf{H}$ & $s$ & 0.07 & 1.0 \\
\hline $\mathbf{H}$ & $p$ & 1.5 & 1.08010285 \\
\hline $\mathbf{H}$ & $p$ & 0.5 & 1.59529336 \\
\hline $\mathbf{H}$ & $p$ & 0.25 & 1.0 \\
\hline $\mathbf{H}$ & $p$ & 0.125 & 1.0 \\
\hline $\mathbf{H}$ & $p$ & 0.03125 & 1.0 \\
\hline c.m. & $s$ & 0.25 & 1.0 \\
\hline c.m. & $s$ & 0.05 & 1.0 \\
\hline c.m. & $s$ & 0.01 & 1.0 \\
\hline \multicolumn{4}{|c|}{46 scattering function basis ${ }^{b}$} \\
\hline $\mathbf{H}$ & $p$ & 4.5 & 1.0 \\
\hline $\mathbf{H}$ & $d_{x y}$ & 1.5 & 1.0 \\
\hline $\mathbf{H}$ & $d_{x y}$ & 0.5 & 1.0 \\
\hline $\mathbf{H}$ & $d_{x y}$ & 0.25 & 1.0 \\
\hline c.m. & $s$ & 0.002 & 1.0 \\
\hline c.m. & $p$ & 0.2 & 1.0 \\
\hline
\end{tabular}

${ }^{a}$ In addition to the $3 s$ contracted $\mathbf{H}$ basis of Ref. 22 .

${ }^{b}$ In addition to the 30 scattering function basis.

tails of these basis sets are given in Table I; the 30- and 46-function sets are contractions of subsets of the basis used in Ref. 11. Examination of Figs. 3 and 4 indicates that, using numerical quadrature of the $V G_{P}^{(+)} V$ matrix elements, the basis of 17 scattering orbitals is already sufficient to give a qualitatively correct $a^{3} \Sigma_{g}^{+}$differential cross section, while improving the basis quickly leads to convergence. Indeed, the residual differences between the 46-function cross section and that of Ref. 11 are sufficiently minor that it is hard to say which is the more nearly converged result; furthermore, some part of these differences may well be due to variations among the basis sets in the description of the target rather than the scattering function, and thus not directly related to the performance of the SMC method.

Similar behavior is found for the $b^{3} \Sigma_{u}^{+}$excitation process, as shown in the differential cross sections of Figs. 5 and 6, again at 20 and $30 \mathrm{eV}$ impact energy. Although in this case the 17-function cross section departs significantly from the converged result at higher scattering angles, it does correctly indicate the qualitative nature of the angular dependence. As was true for the $a^{3} \Sigma_{g}^{+}$channel, the 30- and 46-function results are seen to be of a

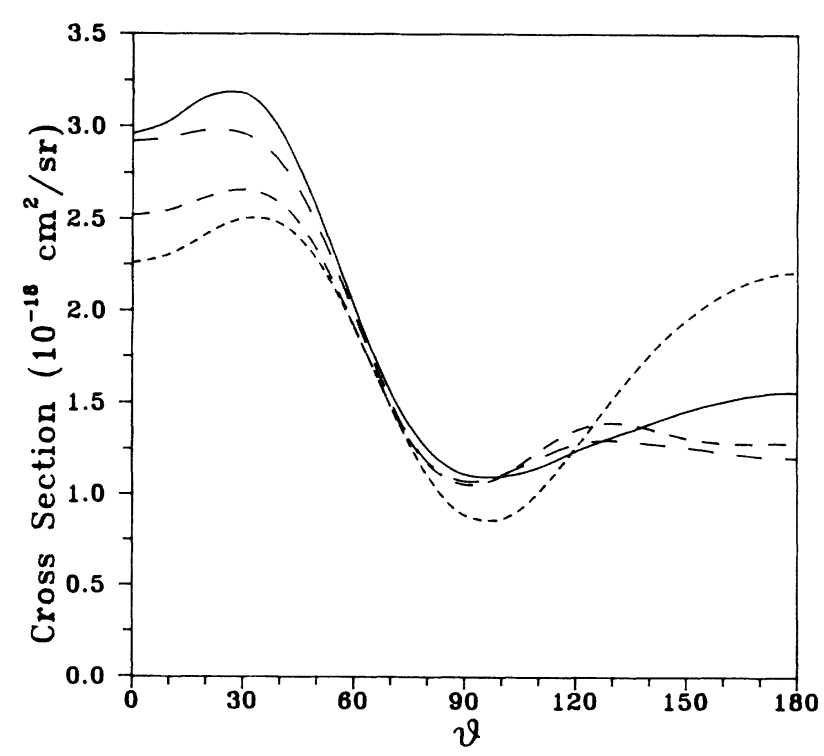

quality comparable to that obtained from a 63-function basis with the insertion procedure. Together, these calculations suggest that the numerical quadrature procedure should make it possible to perform exploratory calculations quite economically on many electronic excitation processes of interest, with quantitatively reliable follow-up studies requiring only modest basis sets.

\section{DISCUSSION AND CONCLUSIONS}

We have discussed a recently developed technique for improving the efficiency of electron-molecule scattering calculations using the SMC method. ${ }^{1-3}$ This improve-

FIG. 6. As in Fig. 4, for the $b^{3} \Sigma_{u}^{+}$state. 
ment consists in replacing the insertion of a Gaussian basis set on either side of the principal-value part of the Green's function with direct numerical quadrature in momentum space. We generally find in practice that the reduction in size of the Gaussian basis which it permits more than compensates for the additional numerical effort of the quadrature. This improved procedure has the further advantage that it allows economical testing for convergence of the $V G_{P}^{(+)} V$ terms. While further improvements are planned, including an investigation of the optimization of the quadrature, the results of the test calculations presented here and those of other studies now under way indicate that, with numerical quadrature of the $V G_{P}^{(+)} V$ integrals, the SMC method can be both an efficient and a reliable tool for the study of electronmolecule collision processes.

\section{ACKNOWLEDGMENTS}

This work was supported by the National Science Foundation under Grant No. PHY-860242 and by the Army Research Office under Contract No. DAAL03-86K-0140. M.A.P.L., L.M.B., and V.M. also acknowledge support by the Conselho Nacional de Pesquisa e Desenvolvimento Científico e Tecnológico (Brazil) and the National Science Foundation through the U.S.-Brasil Scientific Cooperation Program. We also acknowledge use of resources of the San Diego SuperComputer Center, which is supported by the National Science Foundation.
${ }^{1}$ K. Takatsuka and V. McKoy, Phys. Rev. A 24, 2473 (1981).

${ }^{2}$ K. Takatsuka and V. McKoy, Phys. Rev. A 30, 1734 (1984).

${ }^{3}$ See also M. A. P. Lima and V. McKoy, Phys. Rev. A 38, 501 (1988).

${ }^{4}$ T. L. Gibson, M. A. P. Lima, K. Takatsuka, and V. McKoy, Phys. Rev. A 30, 3005 (1984).

${ }^{5}$ W. M. Huo, T. L. Gibson, M. A. P. Lima, and V. McKoy, Phys. Rev. A 36, 1632 (1987); 36, 1642 (1987).

${ }^{6}$ M. A. P. Lima, T. L. Gibson, W. M. Huo, and V. McKoy, Phys. Rev. A 32, 2696 (1985).

${ }^{7}$ M. A. P. Lima, K. Watari, and V. McKoy, Phys. Rev. A 39, 4312 (1989).

${ }^{8}$ L. M. Brescansin, M. A. P. Lima, T. L. Gibson, V. McKoy, and W. M. Huo, J. Chem. Phys. 85, 1854 (1986).

${ }^{9}$ H. P. Pritchard, M. A. P. Lima, and V. McKoy, Phys. Rev. A 39, 2392 (1989).

${ }^{10}$ W. M. Huo, Phys. Rev. A 38, 3303 (1988).

${ }^{11}$ M. A. P. Lima, T. L. Gibson, V. McKoy, and W. M. Huo, Phys. Rev. A 38, 4527 (1988), and references therein.
${ }^{12}$ A. J. R. da Silva, M. A. P. Lima, L. M. Brescansin, and V. McKoy, Phys. Rev. Lett. (unpublished).

${ }^{13}$ See, for example, D. K. Watson, R. R. Lucchese, V. McKoy, and T. N. Rescigno, Phys. Rev. A 21, 738 (1980).

${ }^{14} \mathrm{~K}$. Watari (unpublished).

${ }^{15}$ D. A. Levin, A. W. Fliflet, M. Ma, and V. McKoy, J. Comput. Phys. 28, 416 (1978).

${ }^{16}$ P. McNaughten and D. G. Thompson, J. Phys. B 21, L703 (1988).

${ }^{17}$ C. W. McCurdy and T. N. Rescigno, Phys. Rev. A 39, 4487 (1989).

${ }^{18}$ K. L. Baluja, C. J. Noble, and J. Tennyson, J. Phys. B 18, L851 (1985).

${ }^{19}$ B. I. Schneider and L. A. Collins, J. Phys. B 18, L857 (1985).

${ }^{20}$ T. H. Dunning, J. Chem. Phys. 58, 1017 (1973).

${ }^{21}$ H. Tanaka, T. Okada, L. Boesten, T. Suzuki, T. Yamamoto, and M. Kubo, J. Phys. B 15, 3305 (1982).

22 J. Pacansky and M. Dupuis, J. Chem. Phys. 68, 4277 (1978). 